\title{
Measuring conductivity, temperature and hydrogen peroxide concentration using a single sensor structure
}

\author{
G.R. Langereis, W. Olthuis and P. Bergveld \\ MESA Research Institute, University of Twente \\ P.O. Box 217, 7500 AE Enschede, The Netherlands
}

\section{SUMMARY}

Using a single multi purpose sensor structure, consisting of metal films on a substrate only, three electrolyte parameters have been measured. The parameters are temperature, conductivity and hydrogen peroxide concentration, where the latter one represents one example of many species that can be determined amperometrically. The active area of the fabricated devices is about $1 \mathrm{~mm}^{2}$.

Keywords: Sensor array, conductivity, amperometry.

\section{INTRODUCTION}

Many sensor designers aim at a single, highly selective structure consisting of a selector part and a transducer part. An alternative approach is the use of an array of less-selective simple structures where an intelligent control system is used to determine a set of parameters from a number of experiments

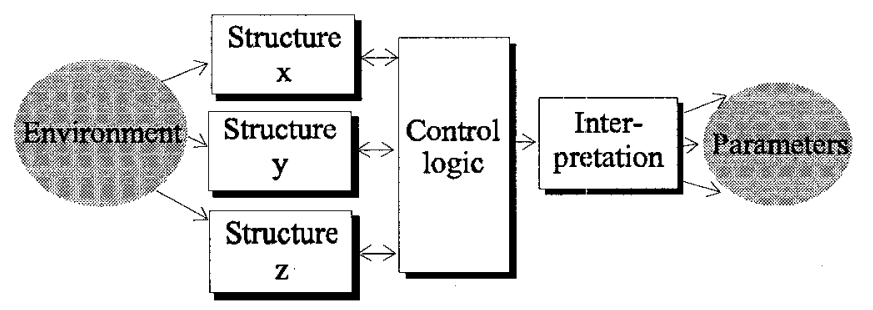

Figure 1: General structure of a sensor array based measurement system

carried out using these structures. Figure 1 shows the general structure of such an array-based measurement system. The multi purpose sensor structure described here is an integration of a number of sensor structures, and can therefore replace a row of separate structures. In terms of figure 1: the multi purpose sensor structure can be used as either structure $\mathrm{x}, \mathrm{y}$ or $\mathrm{z}$ when suitably scheduled by the control logic.

An advantage of performing several measurements using a single sensor structure is that the information certainly comes from the same location in the sample solution. Besides that, the number of contact leads is reduced and the size of the single multi purpose sensor structure will be smaller than three separate structures.

The used structure, as shown in figure 2, consists only of shaped platinum films on a substrate. Its shape is based on an interdigitated structure which is a common design for conductivity sensors. In order to create a resistive path for temperature measurements, the fingers were cut along. With all of the pads in parallel, the structure is a working electrode for amperometric measurements. Notice that for the latter mode of operation an external counter electrode is necessary.

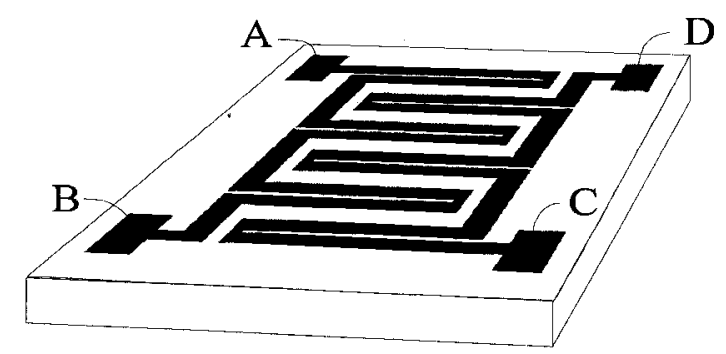

Figure 2: Shape of the multi purpose sensor structure Temperature between pad $A$ and $B$,

Conductivity between pads $A, B$ and $C, D$, Amperometric working electrode with pads $A, B, C$, and D short circuited.

Two implementations of the structure were realised and tested, differing in the number and width of the fingers. They will be referred to as type I and II. Table 1 gives the dimensions for the two implementations of the sensor structure.

Table 1: Device dimensions for the integrated sensor structures

\begin{tabular}{lll}
\hline & Type I & Type II \\
\hline Conductivity & & \\
Space between fingers $[\mu \mathrm{m}]$ & 5 & $15(10)$ \\
Width of the fingers $[\mu \mathrm{m}]$ & 200 & 100 \\
Length of the fingers $[\mu \mathrm{m}]$ & 1025 & 1035 \\
Number of fingers & 5 & 9 \\
\hline Temperature & & \\
Length of the resistive path $[\mathrm{mm}]$ & 10.25 & 8.28 \\
Width of the resistive path $[\mu \mathrm{m}]$ & 47 & 45 \\
\hline Working electrode $\left[\mathrm{mm}^{2}\right]$ & $2 \times 0.48$ & $2 \times 0.42$ \\
\hline Size of structure $\left[\mathrm{mm}^{2}\right]$ & $1.0 \times 1.0$ & $1.0 \times 1.0$ \\
& & \\
\hline
\end{tabular}

\section{THEORY}

The three basic operational modes of the structure, temperature, conductivity and amperometry are selected by choosing the proper connecting pads and applying a specific stimulus to these pads. The parameter to be measured then follows from the voltage-current relation. 


\section{Temperature}

The structure of figure 2 has two resistive paths: between pads $A$ and $B$ and between pads $C$ and $D$. These can be taken separately or serially connected. The resistance of such a metal strip is:

$$
\mathrm{R}_{0}=\frac{\rho \mathrm{l}}{\mathrm{wh}}
$$

with $w$ the width, $h$ the thickness and $l$ the length of the strip and $\rho$ the specific resistivity of the metal. For platinum, the resistivity at room temperature is $1.0 \cdot 10^{-5} \Omega \cdot \mathrm{cm}$. The used structures have an intended thickness of $250 \mathrm{~nm}$. The lengths of the resistive paths are 10.25 and $8.28 \mathrm{~mm}$ and the widths are 47 and $45 \mu \mathrm{m}$ for type I and II respectively. Therefore, the nominal resistances will theoretically be $87 \Omega$ and $74 \Omega$.

The change in resistance due to temperature variations can be written as:

$$
\mathrm{R}(\mathrm{T})=\mathrm{R}_{\mathrm{Ref}} \cdot\left[1+\alpha\left(\mathrm{T}-\mathrm{T}_{\mathrm{Ref}}\right)\right]
$$

with $\alpha$ the temperature coefficient. This coefficient is found in literature [1] to be $3.92 \cdot 10^{-3}{ }^{\circ} \mathrm{C}^{-1}$ for platinum with a nonlinearity of $0.2 \%$ between $0-100^{\circ} \mathrm{C}$.

To measure the resistance a potential should be applied while measuring the current or vice versa. In order to avoid electrochemical interferences with the electrolyte during measurement, the applied voltage should be low or an AC signal must be taken.

\section{Conductivity}

When inputs A, B and C, D are short circuited, the structure can be used as an interdigitated structure [2]. Figure 3 shows the equivalent circuit for a two-points conductivity cell.

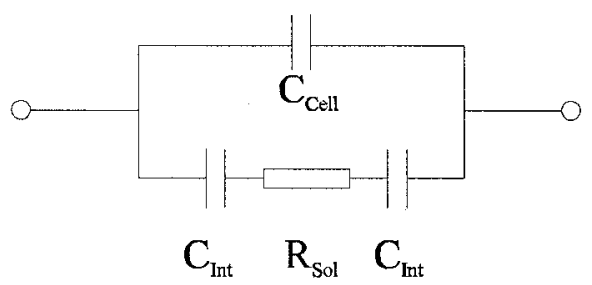

Figure 3: Equivalent circuit for a conductivity cell

The capacities $\mathrm{C}_{\mathrm{Int}}$ represent the metal-electrolyte double layer. Together with the electrolyte resistance $R_{\mathrm{Sol}}$ they cause the first cut-off frequency. Above this frequency, the impedance of the structure is practically equal to the electrolyte resistance. From the electrolyte resistance the conductivity $\kappa_{\text {Sol }}$ can be found using the geometric constant $\mathrm{K}_{\text {Cell }}$ :

$$
\kappa_{\text {Sol }}=\frac{K_{\text {Cell }}}{R_{\text {Sol }}}
$$

The upper limit of the measurement frequency is determined by the cell capacitance $\mathrm{C}_{\text {Cell }}$ or in some cases by the connecting wires parasitic capacitance which is in parallel to $\mathrm{C}_{\mathrm{Cell}}$. The structure was optimised for making the low cut-off frequency as low as possible in order to have a large operational frequency range. The cell constant can theoretically be calculated from the dimensions of the structure [2] and are 2.04 $\mathrm{cm}^{-1}$ for structure I and $1.34 \mathrm{~cm}^{-1}$ for structure II.

\section{Chrono amperometric operation}

As a test system for the amperometric operation of the structure, the oxidation of hydrogen peroxide is taken.

$$
\mathrm{H}_{2} \mathrm{O}_{2} \rightarrow \mathrm{O}_{2}+2 \mathrm{H}^{+}+2 \mathrm{e}^{-}
$$

After applying a positive potential step to an amperometric working electrode, a current response due to the oxidation of hydrogen peroxide can be observed. For a step large enough to deplete hydrogen peroxide at the electrode interface immediately, the current is theoretically described by the Cottrell equation [3]:

$$
\mathrm{i}(\mathrm{t})=\mathrm{nFAC}_{\mathrm{H}_{2} \mathrm{O}_{2}} \sqrt{\frac{\mathrm{D}_{\mathrm{H}_{2} \mathrm{O}_{2}}}{\pi \mathrm{t}}}
$$

with $\mathrm{n}$ the number of electrons transferred, F Faraday's constant, $C$ the bulk concentration and $D$ the diffusion coefficient of hydrogen peroxide. So $\mathrm{i}{ }_{\mathrm{t}}$ yields a factor which is expected to be linearly dependent on the hydrogen peroxide bulk concentration.

With all four pads of the sensor structure short circuited, an amperometric working area of almost one square millimeter is available. This results in a theoretical value for the dependency of $\mathrm{i} V \mathrm{t}$ on the hydrogen peroxide concentration of $2.2 \mu \mathrm{A} V_{\mathrm{sec}}$ per mM.

\section{THE FABRICATION PROCESS}

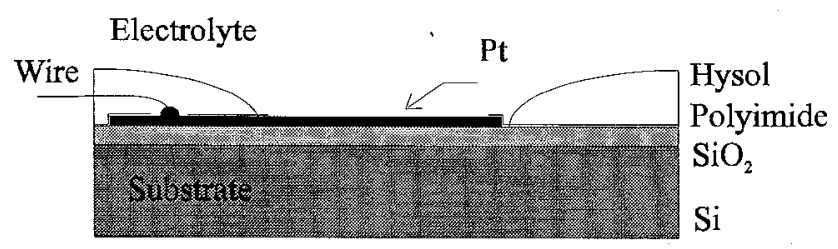

Figure 4: Cross section of the structure

The platinum structure was realised on a silicon substrate with a $1 \mu \mathrm{m}$ thick thermal oxide layer. For electrical insulation of the inactive parts from the solution, a polyimide mask was used. Tantalum layers were used in order to obtain good adhesion between the platinum layer and $\mathrm{SiO}_{2}$ and between platinum and polyimide. The sensors were mounted on a printed circuit board and packaged with Hysol.

\section{EXPERIMENTAL}

The three operational modes were tested sequentially by using advanced laboratory equipment (potentiostat, gain-phase analyser). The development of special control electronics for the optimised use of the structure was left for future work. 


\section{Temperature}

Between pads $A$ and $B$ of the sensor structure a resistance of $85.5 \Omega$ or $178.4 \Omega$ was found for sensor I and II respectively. Although this value is in agreement with the theory for structure $A$, structure $B$ as an $100 \Omega$ offset which can only be explained by a design error in the connecting wires.

An automated set-up was used based on a Radiometer CDM210 conductivity meter in resistance measuring mode.

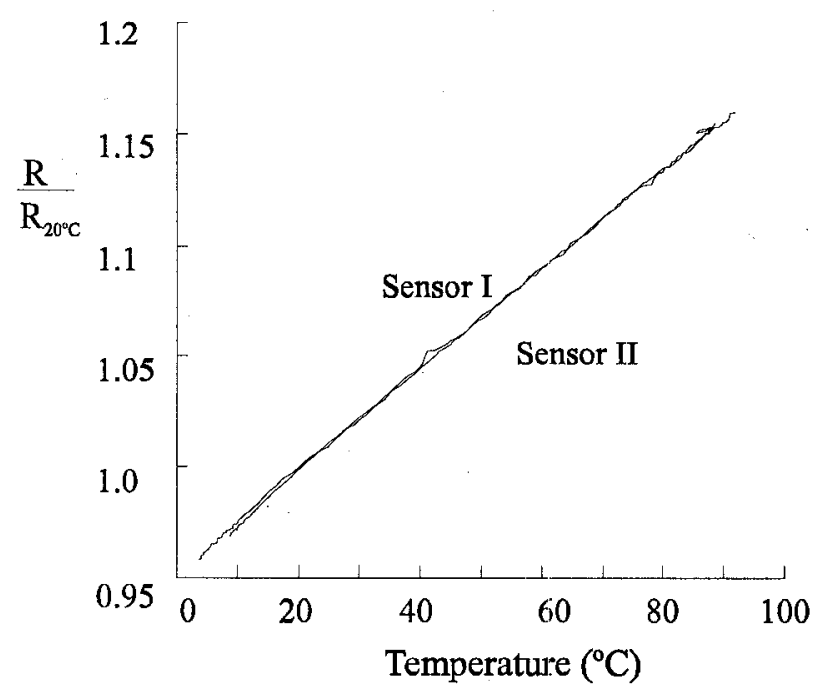

Figure 5: Measured calibration curve for temperature sensing operation

This resulted in a convenient system for recording reproducibly temperature and resistance. The resistance was measured while heating water, starting with melting ice. The normalised temperature scans for sensor I and II are in figure 5. A temperature coefficient of $2.33 \cdot 10^{-3}{ }^{\circ} \mathrm{C}^{-1}$ was found for both types. This value is lower then the one found in literature, probably because the platinum has become an alloy with the tantalum adhesion layers.

\section{Conductivity}

The impedance plots of the sensors at various $\mathrm{KNO}_{3}$ concentrations were determined using a HP4194a gain-phase analyser. The two cut-off frequencies behaviour as predicted in the theory is confirmed by the measurement results presented in figure 6 .

The electrolyte resistance $R_{\text {Sol }}$ can be found from the impedance plot by taking the value of the impedance at the plateau. Equation 3 shows that the conductivity is proportional to the inverse of this resistance. Therefore the admittance at the measured plateau $\left(1 / R_{\mathrm{Sol}}\right)$ is plotted in figure 7 . From the slope of this curve the cell constant can be calculated. This results in values of 1.30 and $1.24 \mathrm{~cm}^{-1}$ for structure I and II respectively. While the second one approaches the theoretical value, the cell constant of structure $I$ is about $10 \%$ lower.

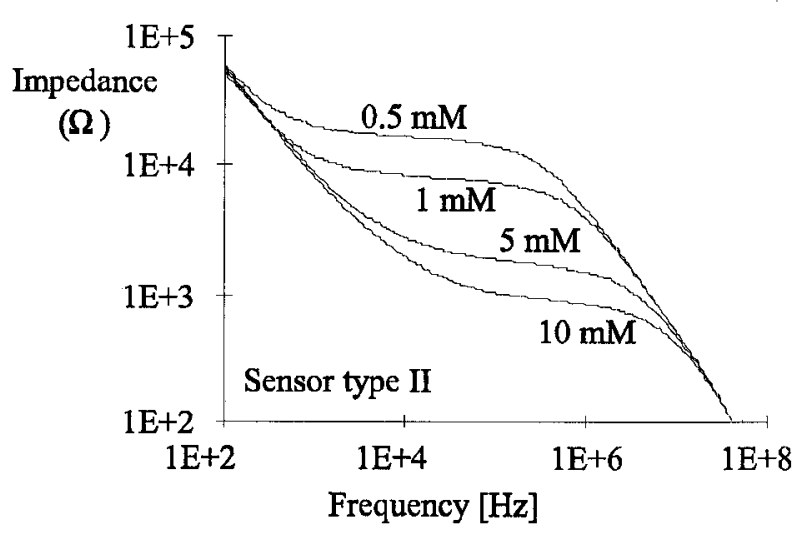

Figure 6: Measured impedance versus frequency of a structure of type $\mathrm{II}_{\text {in }} \mathrm{KNO}_{3}$

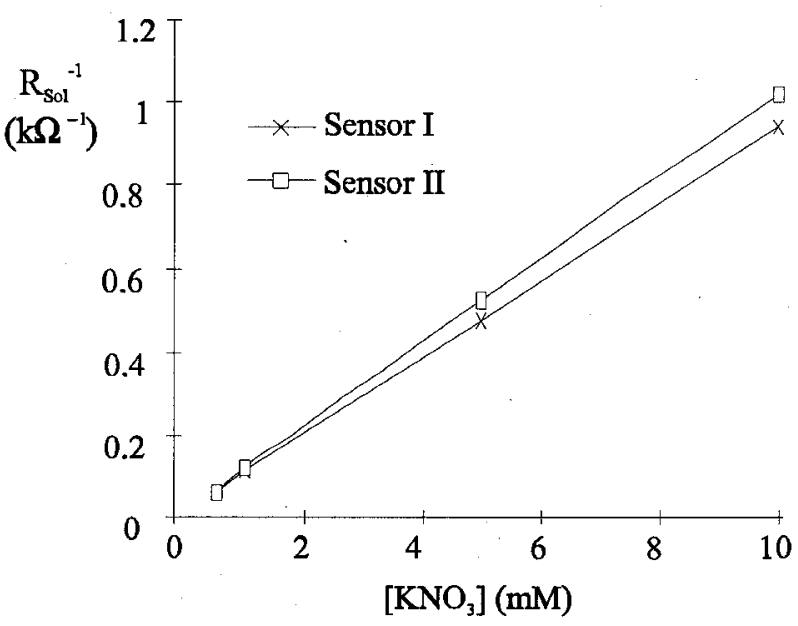

Figure 7: Measured calibration curve for conductivity measurement operation

\section{Hydrogen peroxide concentration}

The structure of figure 2 was used as an amperometric working electrode by connecting inputs $\mathrm{A}, \mathrm{B}, \mathrm{C}$ and $\mathrm{D}$ to the working electrode lead of an EG\&G PAR 173 potentiostat. A three electrode set-up was used with a platinum $5 \times 10 \mathrm{~mm}$ counter electrode and a calomel reference electrode. For the chrono amperometric experiment a potential step of 1 Volt was applied, immediately after diluting to the desired concentration from a $90 \% \mathrm{H}_{2} \mathrm{O}_{2}$ bulk solution. To prevent an ohmic drop across the sample, a background electrolyte of $10 \mathrm{mM} \mathrm{KNO}_{3}$ was used. Figure 8 shows the experimental results for several hydrogen peroxide concentrations.

According to equation 5 , the $\mathrm{i} \sqrt{\mathrm{t}}$ factor is linearly dependent on the bulk hydrogen peroxide concentration. This is confirmed by figure 9 which is constructed from figure 8 by determining the slope between one and five seconds after converting the time axis to $\sqrt{t}^{-1}$. 


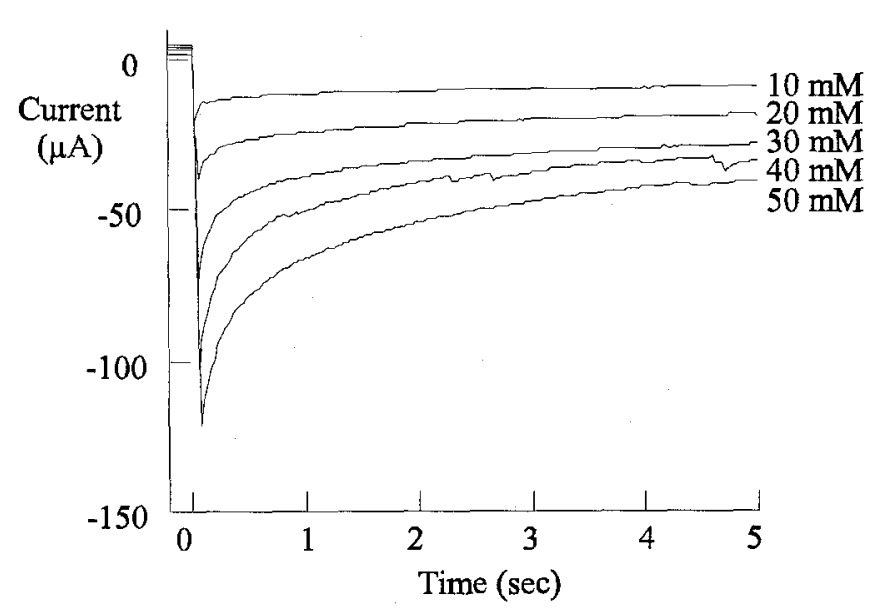

Figure 8: Chrono amperometric experimental results in hydrogen peroxide

Because the active areas of both type devices are almost equal, the slopes in this calibration curves are almost equal as well. The measured slope in figure 9 is about $1.9 \mu \mathrm{A} \sqrt{\mathrm{sec}}$ per $\mathrm{mM}$ $\mathrm{H}_{2} \mathrm{O}_{2}$, which is only slightly lower than the theoretical slope.

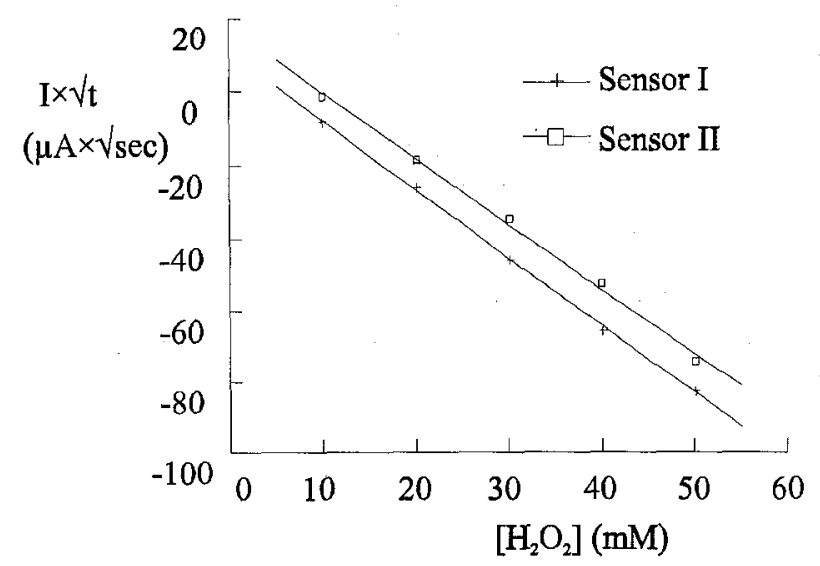

Figure 9: Calibration curve for amperometric detection operation of $\mathrm{H}_{2} \mathrm{O}_{2}$

It will be clear that other redox couples can also be measured in this way.

\section{CONCLUSIONS AND FUTURE WORK}

Using the proposed multi purpose sensor structure, three parameters were successfully measured. Although the structure was realised on a silicon wafer, it can be fabricated using the less expensive thick film technology as well. The integrated structure yields the possibility of very local measurements because the active sensing area is reduced to about $1 \times 1 \mathrm{~mm}^{2}$. The three sensor operations were obtained by connecting the structure to advanced laboratory equipment. In future a dedicated control box will be constructed which schedules these operational modes sequentially or simultaneously.

Besides the three basic sensing operational modes, two possible actuator operations are feasible. First the dissipated energy in the thermoresistive path can be used to heat the local environment of the structure. The second actuator mode is the electrochemical control of the local $\mathrm{pH}$ by the oxidation or reduction of water. Together with the sensor modes this gives a real integrated sensor-actuator device with which many local experiments can be performed in order to find more parameters.

\section{ACKNOWLEDGEMENT}

This research project is financially supported by Unilever.

\section{REFERENCES}

[1] David R. Lide, "Handbook of chemistry and physics", 74 ${ }^{\text {th }}$ edition 1993-1994, CRC Press Inc.

[2] P. Jacobs, A. Varlan, W. Sansen, "Design optimisation of planar electrolytic conductivity sensors", Medical \& Biological Engineering \& Computing, November 1995.

[3] A.J. Bard and L.R. Faulkner, "Electrochemical methods: fundamentals and applications", John Wiley \& Sons, New York, 1980. 